OPEN ACCESS

Edited by:

Gkikas Magiorkinis, National and Kapodistrian University

of Athens, Greece

Reviewed by:

Tara Patricia Hurst, Abcam, United Kingdom

Timokratis Karamitros, University of Oxford, United Kingdom

${ }^{*}$ Correspondence: Thomas Mueller thomas.mueller@medizin.uni-halle.de

Specialty section This article was submitted to Virology, a section of the journal Frontiers in Microbiology

Received: 28 February 2018 Accepted: 23 May 2018

Published: 15 June 2018

Citation: Mueller T, Hantsch C, Volkmer I and Staege MS (2018) Differentiation-Dependent Regulation of Human Endogenous Retrovirus K Sequences and Neighboring Genes in Germ Cell Tumor Cells.

Front. Microbiol. 9:1253. doi: 10.3389/fmicb.2018.01253

\section{Differentiation-Dependent Regulation of Human Endogenous Retrovirus K Sequences and Neighboring Genes in Germ Cell Tumor Cells}

\author{
Thomas Mueller $^{1 *}$, Claudia Hantsch ${ }^{2}$, Ines Volkmer ${ }^{2}$ and Martin S. Staege ${ }^{2}$ \\ ${ }^{1}$ Department of Internal Medicine IV, Haematology/Oncology, Martin Luther University Halle-Wittenberg, Halle, Germany, \\ ${ }^{2}$ Department of Surgical and Conservative Paediatrics and Adolescent Medicine, Martin Luther University Halle-Wittenberg, \\ Halle, Germany
}

Under physiological conditions, most human endogenous retroviruses (HERVs) are transcriptionally silent. However, re-activation of HERVs is observed under pathological conditions like inflammation or cancer. In addition to expression of HERV sequences, an impact of HERV-loci on expression of adjacent genes has been suggested as probably important patho-physiological mechanism. A candidate for such a gene is $\mathrm{PRODH}$ (proline dehydrogenase 1), which is located on chromosome 22 adjacent to HERVK-24. Germ cell tumors (GCTs) are known to express high level of HERVK sequences. In addition, non-seminomatous GCT are useful models to study HERV expression in the context of differentiation since they reflect aspects of cellular development during embryogenesis and usually contain different cell types. This is due to the embryonal carcinoma (EC) cells, which are the stem cell component of GCT. They are pluripotent, show high expression of pluripotency markers like OCT4 and LIN28A and can differentiate into either somatic derivatives (teratoma cells) or choriocarcinoma or yolk-sac tumor cells reflecting extra-embryonal differentiation. OCT4 is lost upon differentiation. We used GCT derived cell lines of varying differentiation stages to analyze expression of HERVK and PRODH. Differentiation status and cellular relationship of GCT cells was determined using microarray analysis and western blotting of the embryonic pluripotency markers OCT4 and LIN28A. The highest expression of HERVK was found in undifferentiated EC cells, which retain a stem cell phenotype and express both OCT4 and LIN28. In contrast, the lowest expression of HERVK was observed in somatic differentiated GCT cells which also lack OCT4 and LIN28A whereas GCT cells with differentiation characteristics of yolk-sac tumor expressed LIN28A but not OCT4 and showed intermediate level of HERVK. A similar pattern was found for PRODH. Differentiation of EC cells by siRNA mediated knock-down of OCT4 or treatment with differentiation inducing medium decreased expression of HERVK and PRODH. Treatment of differentiated GCT cells with $5^{\prime}$-azacytidine and 
trichostatin A increased expression of HERVK and $\mathrm{PRODH}$, indicating that epigenetic mechanisms are responsible for altered expression of these genes. Our data suggest that HERVK expression is dependent on cellular differentiation stages regulated by epigenetic mechanisms, which can also affect expression of neighboring genes.

Keywords: endogenous retroviruses, germ cell tumor, differentiation, HERVK, PRODH, OCT4, LIN28A

\section{INTRODUCTION}

Human endogenous retroviruses (HERVs) are retroviral sequences that are permanently integrated into the human DNA and that are inherited from parents to the offspring like other genes. In addition to HERVs that are present in every individual, some HERVs are polymorphic and the presence or absence of these HERVs varies between individuals (Turner et al., 2001). Interestingly, these HERVs are particularly able to produce virus like particles (Boller et al., 2008). Re-activation of HERVs has been found in cancer patients. The patho-physiological function of this phenomenon is unclear but oncogenic transformation of cells by HERV gene products has been described (Boese et al., 2000; Galli et al., 2005; Argaw-Denboba et al., 2017; Lemaître et al., 2017). In addition, re-activation of HERV-like promoters has been shown to be involved in the aberrant expression of transformation associated genes in lymphoma cells (Lamprecht et al., 2010). Therefore, an impact of HERV-loci on expression of adjacent genes can be suggested as one probably important patho-physiological mechanism.

In this study we focused on a specific HERVK locus, which is referred to as ERVK-24 according to the nomenclature from Mayer et al. (2011). Formerly, this locus was described as HERVK101 (Barbulescu et al., 1999) and c22_A (Ruprecht et al., 2008). ERVK-24 is located on chromosome 22 between the loci for proline dehydrogenase 1 (PRODH) and DiGeorge critical region 5 (DGCR5). DGCR5 has been identified as chromosomal breakpoint in patients with DiGeorge syndrome (Sutherland et al., 1996). As DGCR5 did not contain a functional open reading frame, it was suggested that expression of DGCR5 might reflect a particular chromatin configuration that is required for regulation of adjacent genes (Sutherland et al., 1996). One candidate for such a gene is $P R O D H$. $P R O D H$ is an evolutionarily conserved gene and a homolog of the Drosophila gene sluggish A (Gogos et al., 1999). Like PRODH, sluggish A is a mitochondrial protein and is involved in glutamate synthesis (Hayward et al., 1993). Mutations in $P R O D H$ are a cause of hyperprolinemia and a risk factor for schizophrenia (Bender et al., 2005).

ERVK-24 belongs to a group of HERVs with high expression in patients with germ cell tumors (GCTs) that are positive for antibodies against HERV-proteins (Flockerzi et al., 2008). It seems to be one of the transcriptionally most active HERV in GCT cells (Ruprecht et al., 2008). In addition to their high expression of HERVK sequences, GCTs, in particular non-seminomatous GCTs are useful models to study HERV expression in the context of differentiation processes since they can reflect some aspects of cellular development during embryogenesis. This is due to the pluripotent nature of embryonal carcinoma (EC) cells, which are the stem cell component of GCT. EC cells can be considered as the malignant counterpart of pluripotent embryonic stem cells, and show high expression of pluripotency markers like OCT4 (Looijenga et al., 2003; Sperger et al., 2003). They can differentiate into either somatic derivatives leading to teratoma tissue or into tissues like choriocarcinoma and yolk sac tumor reflecting an extra-embryonic differentiation (Oosterhuis and Looijenga, 2005). OCT4 is lost during differentiation. Therefore, GCT are usually composed of undifferentiated EC cells and variously differentiated cell types (Oosterhuis and Looijenga, 2005).

In the present paper we analyzed expression of HERVK and PRODH in cell lines of GCT with varying differentiation stages and upon induction of differentiation in undifferentiated cells. In addition, differentiated cells were treated with agents modifying DNA methylation and histone acetylation to investigate epigenetic mechanisms, which are known to be involved in both differentiation processes and inactivation of HERVs.

\section{MATERIALS AND METHODS}

\section{Cell Lines and Cell Culture}

The following human GCT cell lines were used: H12.1 and H12.5 (Casper et al., 1987), H12.1D (Mueller et al., 2006), 1411HP (Vogelzang et al., 1985), GCT72 and GCT27 (Pera et al., 1987), 1777NRpmet, 2102EP, 833K, and NTera2-D1 (Bronson et al., 1980, 1983; Andrews et al., 1996). The cell lines 1777NRpmet, $1411 \mathrm{HP}$, and $833 \mathrm{~K}$ were kindly provided by Prof. Peter W. Andrews (University of Sheffield, United Kingdom). The H12.1 and H12.5 were established in the former group of Prof. H.-J. Schmoll (University Hospital Halle, Germany) and belong to our lab. The cell lines GCT72 and GCT27 were kindly provided by Prof. Martin F. Pera (Monash University, Australia, at the time of shipping). The NTera2-D1 was kindly provided by Dr. Heiko van der Kuip (University of Tübingen, Germany).

The Hodgkin lymphoma (HL) cell lines L-1236, L-428, L-540, KM-H2, and HDLM-2 (Schaadt et al., 1979; Diehl et al., 1982; Drexler et al., 1986; Kamesaki et al., 1986; Wolf et al., 1996) were purchased from the German Collection of Microorganisms and Cell Cultures, Brunswick, Germany.

All cell lines were cultured in RPMI-1640 (Invitrogen, Karlsruhe, Germany) supplemented with $10 \%$ fetal calf serum, $100 \mathrm{U} / \mathrm{mL}$ penicillin, and $100 \mu \mathrm{g} / \mathrm{mL}$ streptomycin at $37^{\circ} \mathrm{C}$ in a humidified atmosphere with $5 \% \mathrm{CO}_{2}$. For induction of differentiation of H12.1 cells, cells were treated with $10 \mu \mathrm{M}$ retinoic acid and harvested after 5 days. For re-induction of HERVK expression, 1777NRpmet cells were treated with $5^{\prime}$ azacytidine $(4 \mu \mathrm{M})$ and trichostatin $\mathrm{A}(10 \mathrm{nM})$ and harvested after 5 days. 


\section{Gene Expression Analysis}

We used published cell lines and commercially available RNA from anonymous sources for gene expression analysis. RNA from cell lines was isolated using Trizol reagent (Invitrogen, Karlsruhe, Germany) following the manufacturer's protocol. Probable DNA contamination was removed by treatment with DNase (Roche, Mannheim, Germany). In addition, RNA from human placenta from anonymous donors was obtained from Becton-Dickinson (Heidelberg, Germany). RNA (2 $\mu \mathrm{g})$ were transcribed into cDNA using oligo-dT12-18 primers (Promega, Mannheim, Germany) and polymerase chain reaction (RT-PCR) was performed. The following primer combinations were used: actin beta (ACTB): 5'-GGC ATC GTG ATG GAC TCC G-3', 5'-GCT GGA AGG TGG ACA GCG A-3'; HERVK primer combination a (HERVKa): $5^{\prime}$-CCT GCA GTC CAA AAT TGG TT-3', 5'-GCA ATG CAA CTC CTG CTA CA-3'; HERVK primer combination $b\left(\right.$ HERVKb): $5^{\prime}$-TTC TGC TGG TGA GAG CAA GA-3', 5'-TGG ACA CAG CAC ATG TTT CA-3'; glyceraldehyde 3-phosphate dehydrogenase (GAPDH): 5'-CCA TGG AGA AGG CTG GGG-3', 5' -CAA AGT TGT CAT GGA TGA CC-3'; proline dehydrogenase 1 (PRODH): 5'-GAG GCT TTG AGA AGC CAG TG-3', 5' -GGT ATT GCT TGT CCC GCT TA- $3^{\prime}$. The PCR conditions were: $94^{\circ} \mathrm{C}, 30 \mathrm{~s} ; 60^{\circ} \mathrm{C}, 30 \mathrm{~s} ; 72^{\circ} \mathrm{C}$, $45 \mathrm{~s}$ (35 cycles). The HERVK primers bind to the following genome coordinates: HERVKa:NC_000022.11:18945350-18945369 and NC_000022.11:18946285-18946304; HERVKb:NC_000022.11: 18946101-18946120 and NC_000022.11:18947034-18947049. The reverse primer from this combination has two mismatches with the current genome version. The primers should also be able to amplify additional HERVK elements. However, the very high expression of ERVK-24 in comparison to other elements seem to favor amplification of this locus as proved by sequencing of polymerase chain reaction products (see Supplementary Material). Absence of DNA contamination was tested randomly by using RNA without reverse transcription as template for PCR. See the Supplementary Material for an example. PCR products were subjected to agarose gel electrophoresis in the presence of ethidium bromide. Real-time quantitative RT-PCR (qRT-PCR) was performed using the Maxima ${ }^{\text {TM }}$ SYBR Green qPCR Master Mix (Fermentas, Sankt Leon-Rot, Germany) using the following conditions: $94^{\circ} \mathrm{C}, 45 \mathrm{~s} ; 60^{\circ} \mathrm{C}, 45 \mathrm{~s} ; 72^{\circ} \mathrm{C}, 60 \mathrm{~s}(40$ cycles).

Global gene expression in GCT cells was analyzed using Affymetrix HG_U133A arrays (Affymetrix, Santa Clara, CA, United States). Arrays were processed essentially as described (Staege et al., 2004). In short, biotinylated cRNA was prepared by in vitro transcription after synthesis of double-stranded cDNA. After fragmentation of cRNA and hybridization, signals were detected with streptavidin-phycoerythrin and signals were enhanced by using goat-anti-streptavidin antibodies. Arrays were washed and stained with a GeneChip Fluidics Station 400 and scanned with a GeneArray Scanner G2500A. Affymetrix cell files were processed using Robust Multi-array Average (RMA) algorithm with Expression Console 1.1 (Affymetrix). GCT associated genes were identified on the basis of Wilks' Lambda score (WLS) by using MAFilter (Winkler et al., 2012). WLS was used descriptively without significance calculation for filtering probe sets with high signal intensities in GCT cells in comparison to normal cells. For this end, WLS was calculated as quotient of the variance in the total group of samples and the variance in the group of normal tissues alone. Microarray cell files have been submitted to the Gene Expression Omnibus (GEO) data base (Accession No. GSE113423). For comparative analysis, published microarray data from a panel of normal tissues [normal body atlas (NBA)] from the GEO data base (GSE2361) were used (Ge et al., 2005). Cluster analysis

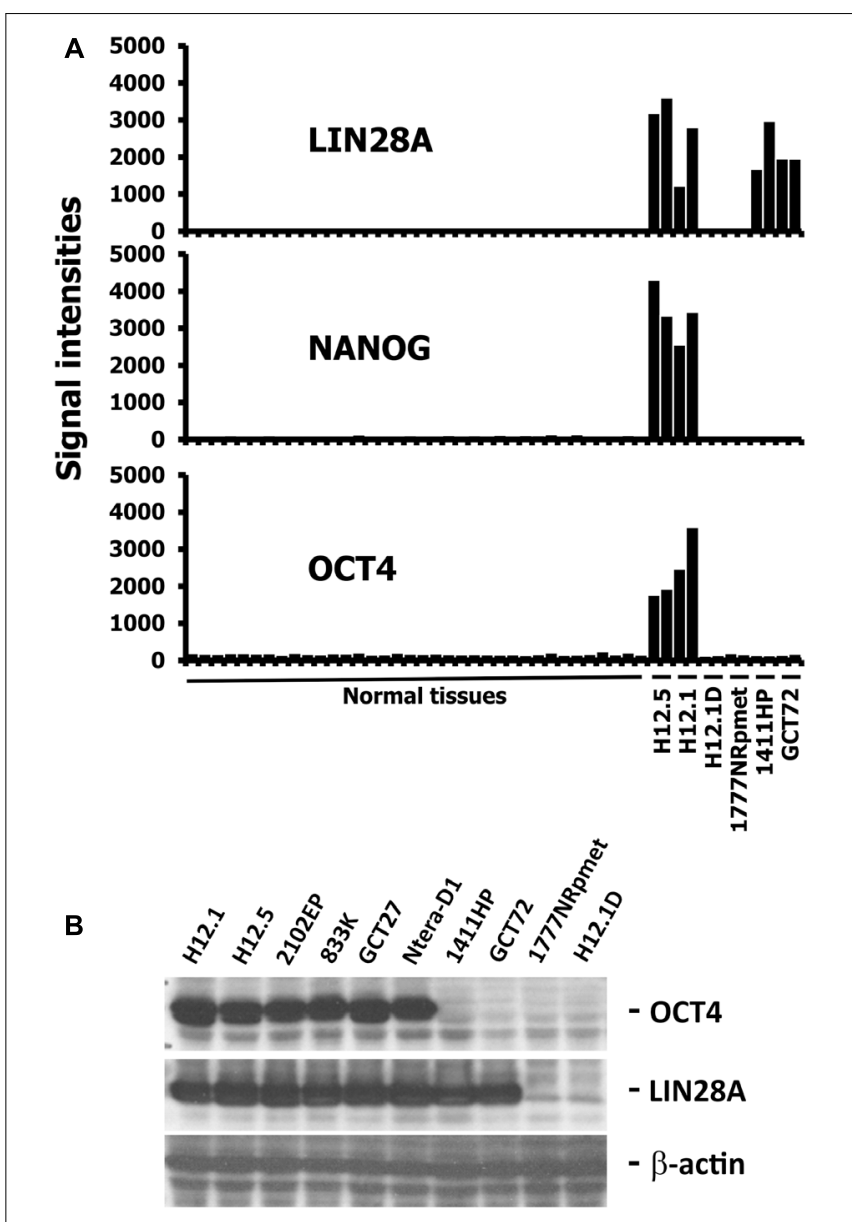

FIGURE 1 | Expression of stem cell markers in GCT cells. (A) Gene expression in GCT cell lines was assessed by DNA microarray analysis. Two independent samples per cell line were analyzed. Gene expression in GCT was compared with gene expression in a panel of normal tissues (Ge et al., 2005). Genes with high expression in GCT were filtered by using MAFilter. Probe sets with a WLS > 10 were considered to be GCT specific. Presented are signal intensities (arbitrary units) for probe sets with specificity for the indicated stem cell markers. The following normal tissues are included (from left to right): heart, thymus, spleen, ovary, kidney, skeletal muscle, pancreas, prostate, small intestine, colon, placenta, bladder, breast, uterus, thyroid, skin, salivary gland, trachea, cerebellum, brain, fetal brain, adrenal gland, bone marrow, amygdala, caudate nucleus, corpus, hippocampus, thalamus, pituitary gland, spinal cord, testis, liver, stomach, lung, fetal lung, fetal liver. (B) Western blot analysis of pluripotent stem cell markers OCT4 and LIN28A. In addition to $\mathrm{H} 12.1$ and $\mathrm{H} 12.5$, four other cell lines representing the undifferentiated, pluripotent EC cell type were analyzed: 2102EP, 833K, GCT27, NTera-D1. 

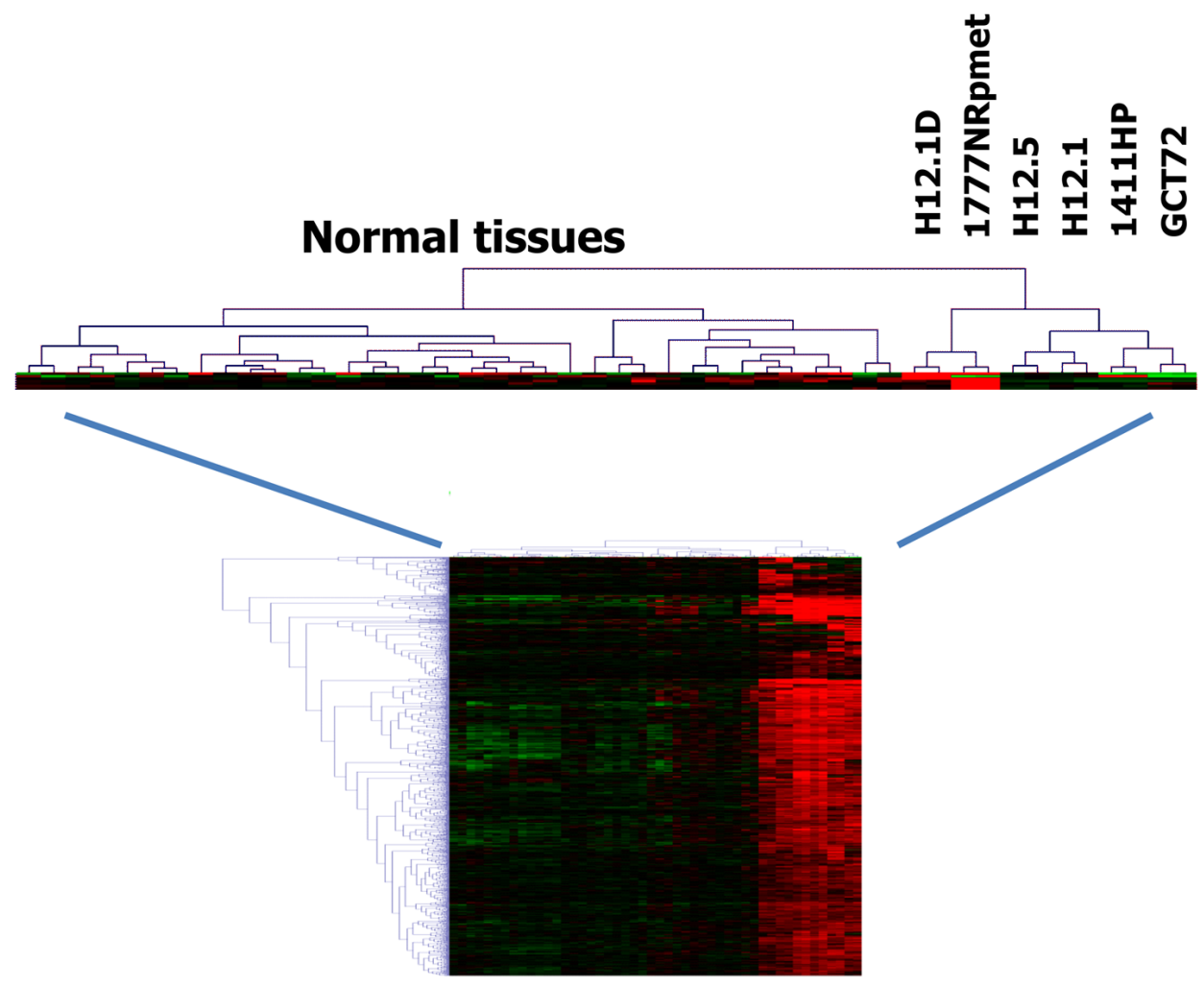

FIGURE 2 | Relationship of GCT cells (i). Gene expression in GCT cell lines was assessed by DNA microarray analysis using two samples per cell line. Gene expression in GCT was compared with gene expression in a panel of normal tissues (Ge et al., 2005). Genes with high expression in GCT were filtered by using MAFilter. Probe sets with a WLS > 10 were considered to be GCT specific. Presented is a cluster analysis using these probe sets. Manhattan distance was used as distance metric. Signal intensities from microarray analysis were log2-transformed and median centered. Green indicates low expression, red high expression.

and visualization was performed with Genesis (Sturn et al., 2002).

\section{Sequencing and Bioinformatical Analyses}

Polymerase chain reaction products were purified with NucleoSpin Gel and PCR Clean-up (Machery-Nagel, Düren, Germany). Sequencing of PCR products was performed using the BigDye Terminator v1.1 Cycle Sequencing Kit (Life Technologies, Austin, TX, United States). The sequences were analyzed with BLAST (Altschul et al., 1990). Open reading frames in the intergenic region between PRODH and DGCR5 were identified by using getorf ${ }^{1}$. Long terminal repeats (LTRs) were identified using RepeatMasker².

\section{Western Blot Analysis}

Cells were harvested by trypsiniziation, rinsed twice with PBS and lysed in RIPA buffer (50 mM Tris- $\mathrm{HCl} \mathrm{pH} \mathrm{8.0,} 100 \mathrm{mM}$ $\mathrm{NaCl}, 0.5 \%$ NP40, 0.5\% DOC, 0.5\% SDS) supplemented with a protease inhibitor cocktail (Sigma, St. Louis, MO, United States). Insoluble components were removed by centrifugation and

${ }^{1}$ http://www.hpa-bioinfotools.org.uk/pise/getorf.html

${ }^{2} \mathrm{http} / / / \mathrm{www} \cdot$ repeatmasker.org/ protein concentrations were measured (BIO-RAD protein assay, Bio-Rad, Hercules, United States). After boiling for $5 \mathrm{~min}$ in SDS-loading buffer (500 mM Tris- $\mathrm{HCl}$ pH 6.8; $10 \%$ glycerol, $2 \%$ SDS, 5\% 2-mercaptoethanol, 0.05\% bromophenol blue), $20 \mu \mathrm{g}$ protein per lane was separated by SDS-PAGE and electroblotted onto nitrocellulose transfer membrane (Whatman, Maidstone, United Kingdom). Equal protein loading was controlled by Ponceau S staining (Sigma, St. Louis, MO, United States). Membranes were blocked with 5\% non-fat dry milk in PBST for $1 \mathrm{~h}$ and probed for $2 \mathrm{~h}$ with the primary antibodies diluted in PBST/5\% milk followed by incubation with secondary HRPconjugated antibodies. Proteins were visualized by enhanced chemiluminescence (Carl Roth, Karlsruhe, Germany). The following primary antibodies were used: OCT4: sc-5279 mouse monoclonal C-10; $\beta$-actin: sc-1615 goat polyclonal C-11 (both from Santa Cruz Biotechnology, Santa Cruz, CA, United States); LIN28A: \#3978 rabbit polyclonal (Cell Signalling). Horseradish peroxidase (HRP)-conjugated anti-goat, anti-mouse and antirabbit IgG (all from Santa Cruz Biotechnology, Santa Cruz, CA, United States) were used as secondary antibodies.

\section{siRNA-Mediated Protein Knock-down}

For siRNA mediated protein knock-down of OCT4, cells were transfected with OCT4-specific siRNA or control-siRNA (both 


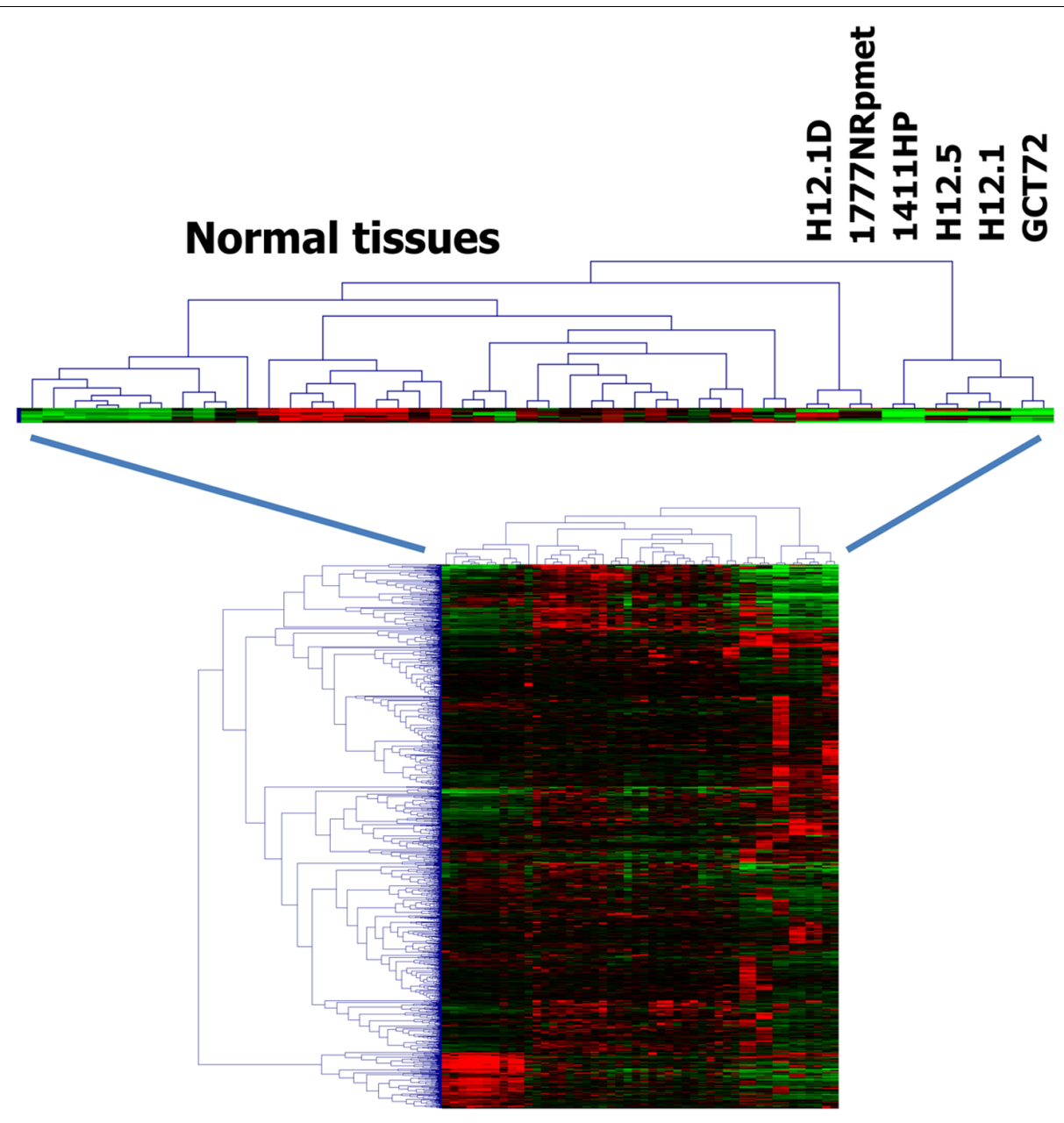

FIGURE 3 | Relationship of GCT cells (ii). Gene expression in GCT cell lines was assessed by DNA microarray analysis using two samples per cell line. Gene expression in GCT was compared with gene expression in a panel of normal tissues (Ge et al., 2005). For each cell line individual specific genes were identified by dividing the mean signal intensity of these cell lines by the 85th percentile of the signal intensities in all other GCT cell lines. Only probe sets that showed a quotient of greater than two were analyzed further. Presented is a cluster analysis. Manhattan distance was used as distance metric. Signal intensities from microarray analysis were log2-transformed and median centered. Green indicates low expression, red high expression.

from Santa Cruz Biotechnology, United States). Transfection of siRNA was performed by the Nucleofector ${ }^{\circledR}$-technology (Amaxa Biosystems, Germany). Cells $\left(2 \times 10^{6}\right)$ were suspended in $100 \mu \mathrm{l}$ transfection buffer (Amaxa Biosystems, Germany) and combined with $1 \mu \mathrm{g}$ siRNA. After reaction in the Nucleofector ${ }^{\circledR}$-system, the transfected cell suspension was diluted in growth media, seeded in 6 well plates and incubated for indicated times. OCT4 knock-down was confirmed by western blot analysis.

\section{RESULTS AND DISCUSSION}

To investigate cellular relationship, we analyzed the gene expression pattern of six GCT cell lines with varying differentiation stages in comparison to a panel of normal tissues (NBA) from the GEO database (Ge et al., 2005). Microarray data were filtered for up-regulated genes on the basis of WLS by using MAFilter and 1,104 probes sets were identified with a WLS $>10$ indicating up-regulation of the corresponding genes in GCT cells. Among the strongest up-regulated genes we found typical markers of pluripotent stem cells like LIN28A, $N A N O G$, and OCT4. Based on the expression pattern of LIN28A, NANOG and OCT4, three groups of GCT cells could be defined (Figure 1A). Group 1 included the cell lines H12.1 and H12.5, which represent the undifferentiated, pluripotent EC cell type. These GCT cells are characterized by the expression of all three genes. Group 2 included the cell lines GCT72 and 1411HP, which have characteristics of differentiation toward yolk-sac tumor. These cells have lost expression of NANOG and OCT4 but still express LIN28A. Group 3 included the cell line 1777NRpmet and the H12.1D, which is an in vitro differentiated, stable derivative of the EC cell line H12.1. These cells have lost expression of all three stem cell markers. The differential gene expression patterns were confirmed by western blot analysis of OCT4 and LIN28A (Figure 1B) indicating that both markers are useful to define the three groups of GCT cells. 


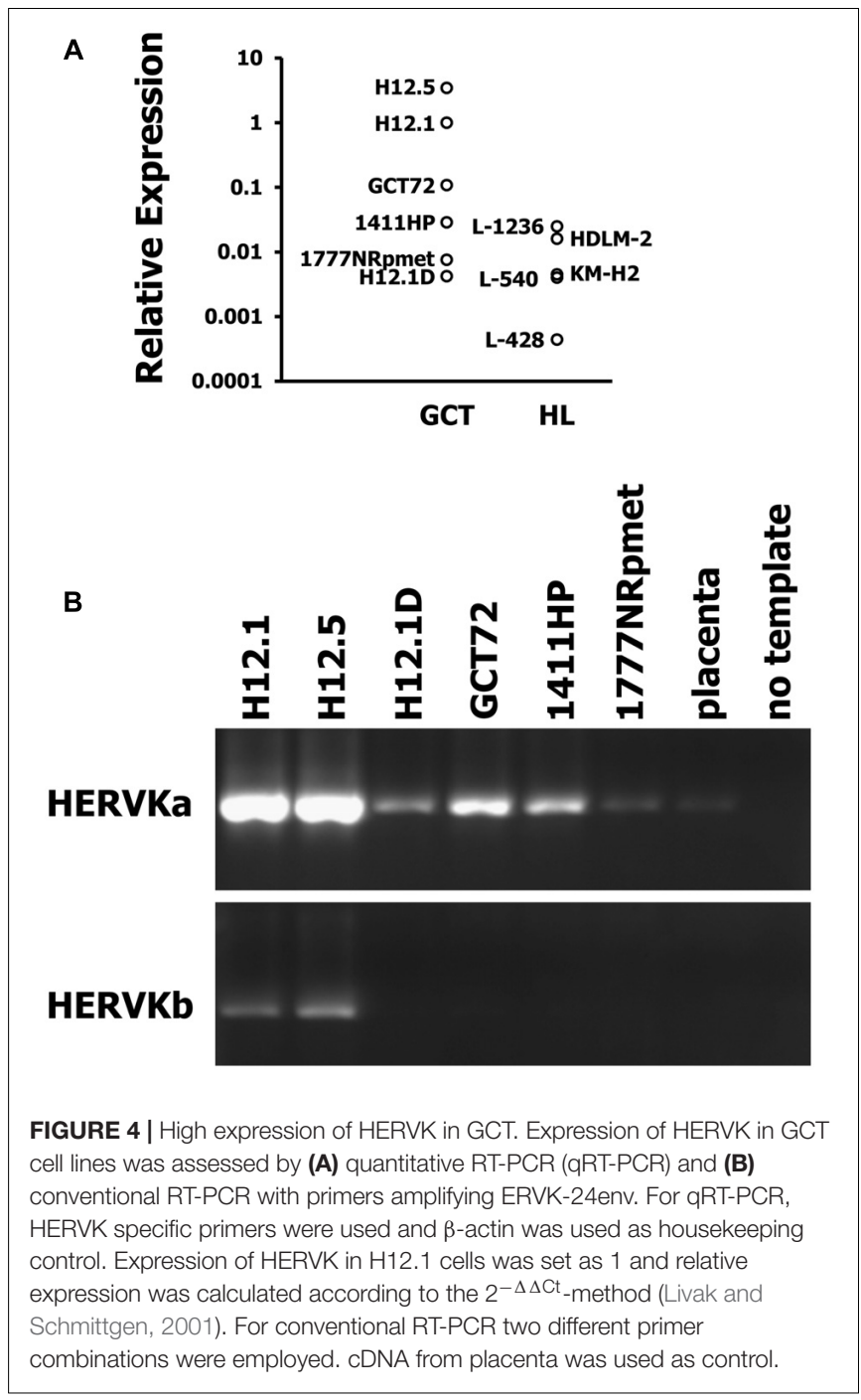

Cluster analysis indicated that the gene expression profile of cells from group 3 have greater similarity with normal somatic tissues than the gene expression profiles of the other groups (Figure 2) indicating a teratoma-like, somatic differentiation lineage of these cells. This similarity could also be seen in cluster analysis when we used probe sets that were filtered for cell linespecificity (Figure 3). For this end, we divided the mean signal intensity of each cell line (which in our case is identical to the 50th percentile) by the 85th percentile of the signal intensities in all other GCT cell lines. Using the 85th percentile has the advantage that outliers from these cell lines have only low impact on the calculated ratios. Cell line specificity was considered if this ratio was greater than 2 . Based on this filtering criterion, a total of 1,315 probe sets showed cell line specificity. Cluster analysis using these probe sets as data points revealed again and more clearly the higher similarity between normal somatic tissues and 1777NRpmet and H12.1D cells. Interestingly, it also revealed that among the cells with yolk-sac tumor characteristics, GCT72 cells are closer related to pluripotent $\mathrm{H} 12.1 / \mathrm{H} 12.5$ cells than $1411 \mathrm{HP}$ cells (Figure 3).
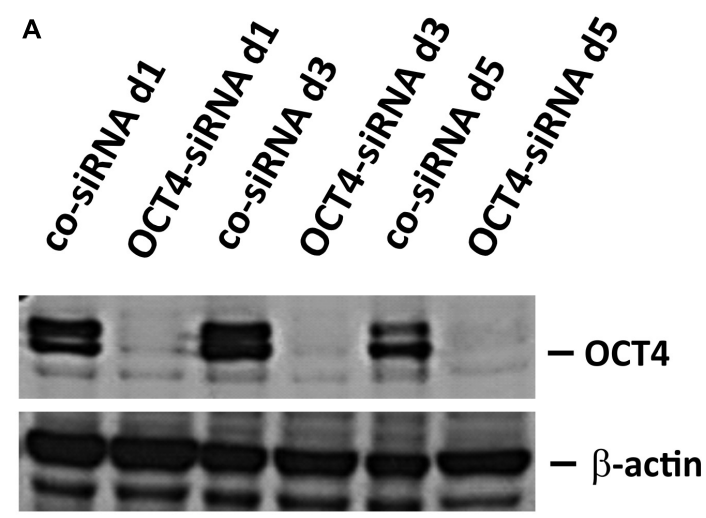

B

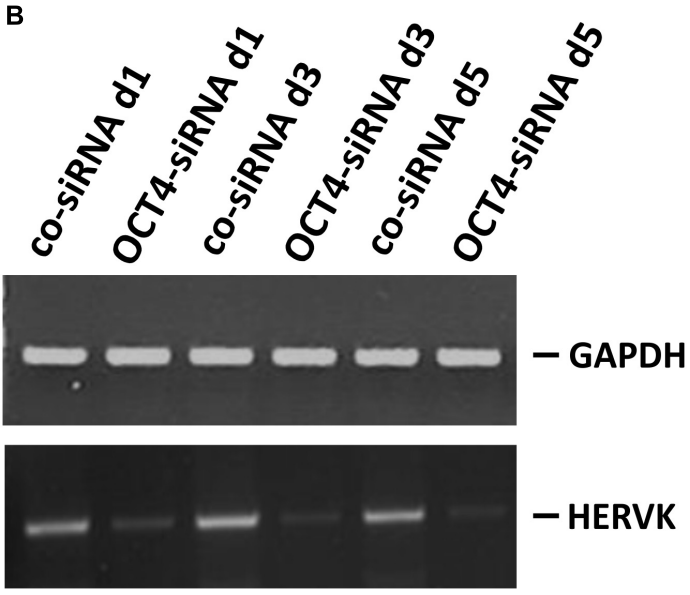

FIGURE 5 | Repression of HERVK upon induction of differentiation in pluripotent GCT cells. Induction of differentiation in pluripotent $\mathrm{H} 12.1$ cells was performed by siRNA mediated knock-down of OCT4. Cells were analyzed after 24, 72, and $120 \mathrm{~h}$ by western blotting to prove knock-down of OCT4 (A) and by RT-PCR for HERVK expression (B).

Together, gene expression analysis and western blotting could define three groups of GCT cells: (i) OCT4+/LIN28+ undifferentiated pluripotent, (ii) OCT4-/ LIN28+ differentiated toward yolk-sac tumor, and (iii) OCT4-/ LIN28- somatic differentiated.

Germ cell tumors are known for their high expression of endogenous retroviruses. Therefore, we tested expression of HERVK in GCT cell lines by conventional and quantitative RT-PCR. For comparison we used HL cell lines. As shown in Figure 4A, GCT cell lines showed higher expression of HERVK than HL cell lines. Although reactivation of HERVs in HL cells has been described, the expression in HL cells is low in comparison to GCT at least for HERVK. Next we asked whether the different differentiation status of our GCT cell lines might have an impact on HERVK expression. Among GCT cells expression of HERVK was particularly high in undifferentiated pluripotent EC cell lines H12.1 and H12.5 (Figure 4B). Cells with characteristics of yolk-sac tumor 
(GCT72 and 1411HP) showed intermediate expression whereas somatically differentiated tumor cells (1777NRpmet, H12.1D) expressed lowest levels (Figure 4B). To prove a direct link between differentiation processes and HERVK expression, we performed siRNA mediated knock-down of OCT4 in pluripotent H12.1 cells which induces differentiation in those cells. As shown

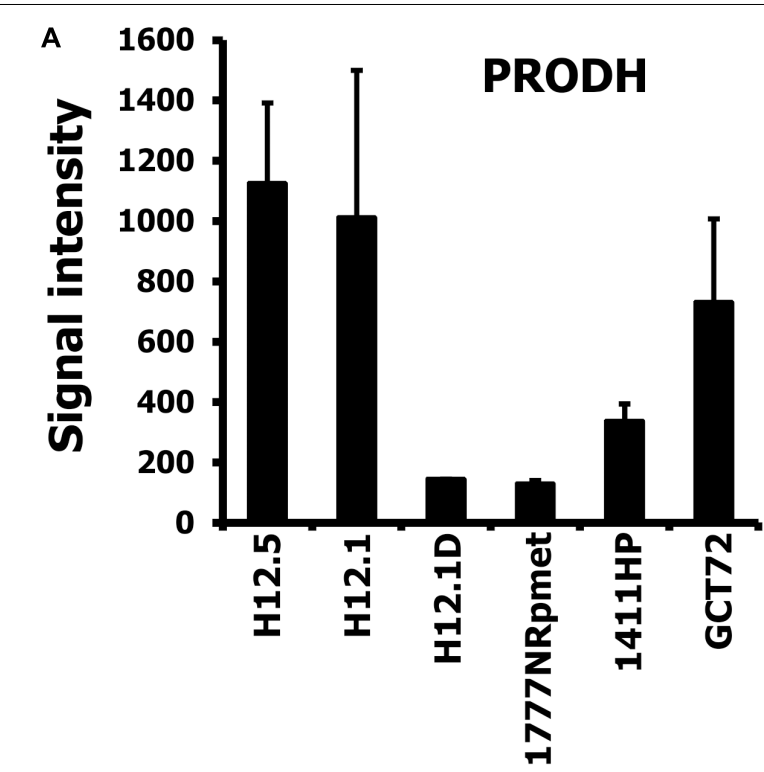

B

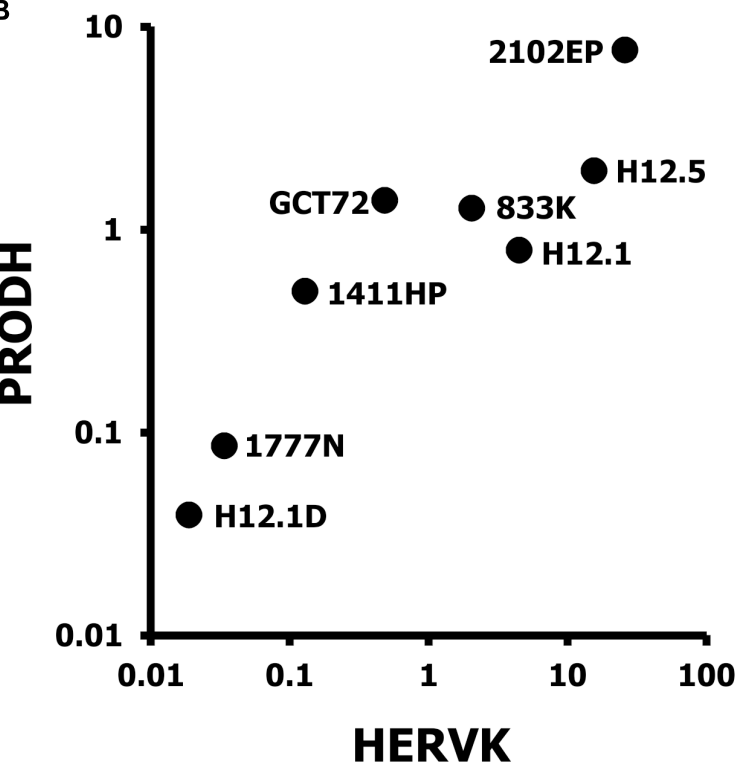

FIGURE 6 | Correlative expression of HERVK and PRODH in GCT cells. (A) Gene expression in GCT cell lines was assessed by DNA microarray analysis. Two independent samples per cell line were analyzed. Presented are signal intensities (arbitrary units) for probe sets with specificity for PRODH as means \pm SD. (B) Presented are results from quantitative RT-PCR analysis with primers specific for HERVK and PRODH. cDNA was prepared from GCT cell lines and $\beta$-actin was used as house-keeping control. Two additional cell lines (2102EP, 833K), which represent the undifferentiated, pluripotent EC cell type were included to support the correlation and to analyze differences among this group of GCT cells. in Figure 5, induction of differentiation rapidly led to repression of HERVK.

Sequencing of PCR products indicated that the primers used for PCR amplify preferentially ERVK-24 (see Supplementary Material). ERVK-24 is located between the loci for PRODH
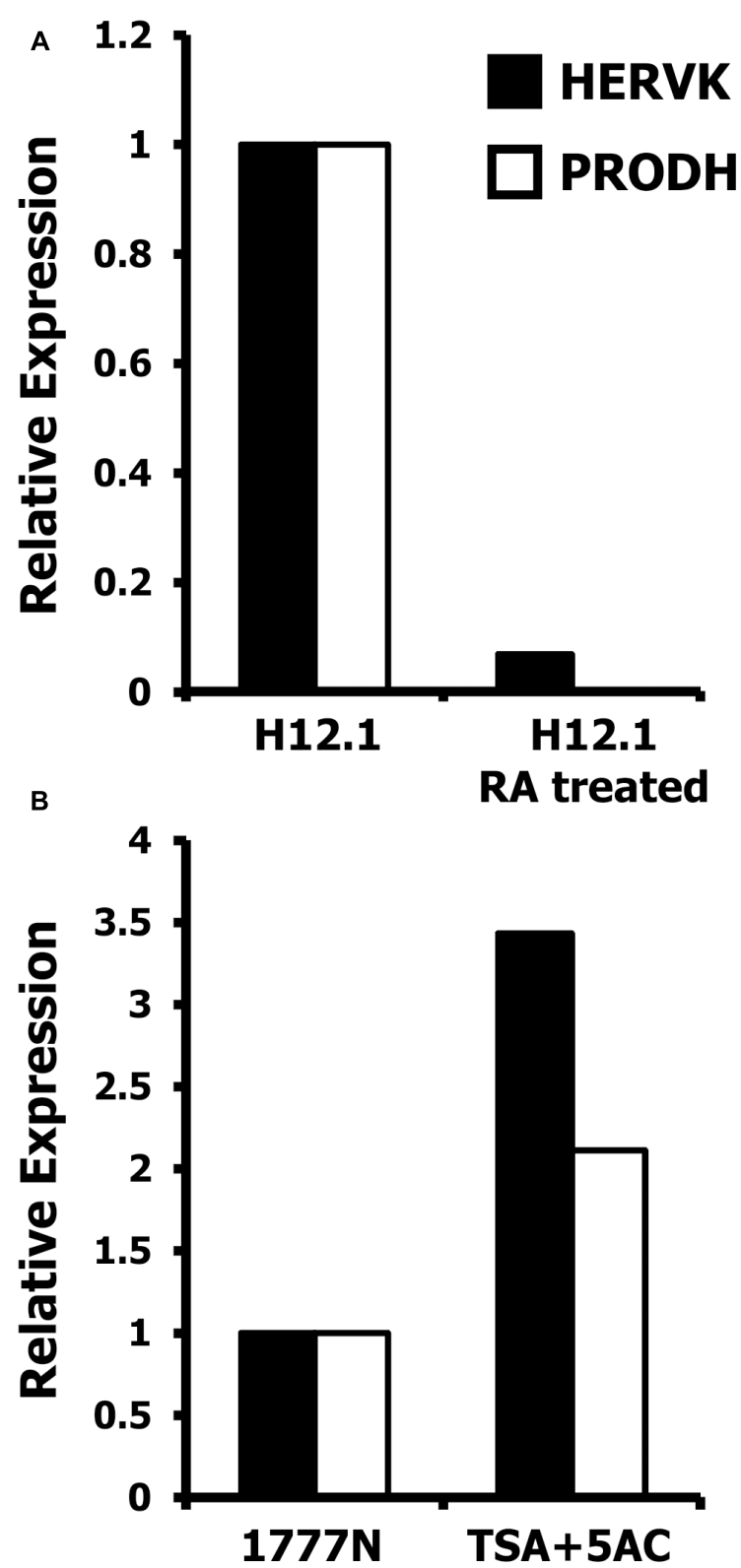

FIGURE 7 | Epigenetic regulation of HERVK and PRODH in GCT cells. Presented are results from quantitative RT-PCR analysis with primers specific for HERVK and PRODH. CDNA was prepared from GCT cell lines and $\beta$-actin was used as house-keeping control. Expression of HERVK in un-treated cells was set as 1 and relative expression was calculated according to the $2^{-\Delta \Delta C t}$-method (Livak and Schmittgen, 2001). (A) Pluripotent H12.1 cells were treated with retinoic acid to induce differentiation. (B) Somatically differentiated 1777 NRpmet cells were treated with a combination of $5^{\prime}$-azacytidine and trichostatin A to induce re-expression of HERVK and $\mathrm{PRODH}$. 
and DGCR5 (see Supplementary Material for the topography of the complete locus including the position of PCR amplicons). Organization of the locus suggests that PRODH and ERVK24 might be regulated by a bi-directional promoter. We asked whether ERVK-24 and the neighboring PRODH might be coregulated. Analysis of PRODH based on our microarray data showed a similar expression pattern of PRODH as observed for HERVK regarding the three groups of GCT cells (Figure 6A). Notably, GCT72 yolk-sac tumor cells had similar high PRODH expression as pluripotent H12.1 cells. Next we performed combined RT-PCR analysis of HERVK and PRODH in our GCT cell panel and found a correlation between PRODH and HERVK expression (Figure 6B). The characteristic higher PRODH expression in GCT72 among the cells with yolk-sac tumor differentiation could be reproduced. Therefore, PRODH and HERVK expression pattern of GCT72 confirmed the cluster analyses and suggest that it is more closely related to pluripotent $\mathrm{H} 12.1 / \mathrm{H} 12.5$ cells than $1411 \mathrm{HP}$.

To further investigate a possible differentiation dependent coregulation of HERVK and PRODH, pluripotent H12.1 cells were treated with differentiation inducing retinoic acid. As shown in Figure 7A, induction of differentiation led to repression of HERVK and was accompanied by down-regulation of PRODH. Next we asked whether HERVK could be re-induced in cells with low expression, e.g., somatic differentiated cells. As shown in Figure 7B, treatment of $1777 \mathrm{NRpmet}$ cells with $5^{\prime}$-azacytidine and trichostatin A increased expression of HERVK. Interestingly, this was accompanied by induction of PRODH expression. Together these data demonstrate a differentiation-dependent and epigenetically-regulated expression of HERVK and suggest coregulation of PRODH expression.

Expression of HERV sequences has been observed in different diseases including cancer. It remains unclear whether HERV expression is directly involved in pathogenesis or whether HERV expression is only an epi-phenomenon of altered gene regulation under pathological conditions. More recently, it was shown that activation of an endogenous retroviral LTR-like promoter is responsible for the expression of growth factor receptors in cancer cells (Lamprecht et al., 2010). However, the reasons for the aberrant activation of such promoters in cancer cells require further investigation. In the present paper we analyzed the expression of the PRODH/ERVK-24 locus. PRODH has been identified as a putative tumor suppressor gene (Liu et al., 2008, 2010). On the other hand, knock-down of PRODH decreases the viability of oxidized low-density lipoprotein (OxLDL)-treated cancer cells (Zabirnyk et al., 2010). OxLDL induce PRODHdependent autophagy which may explain some of the effects of PRODH, because limited autophagy is a cell survival factor whereas excessive autophagy promotes cell death (Degenhardt et al., 2006).

\section{REFERENCES}

Altschul, S. F., Gish, W., Miller, W., Myers, E. W., and Lipman, D. J. (1990). Basic local alignment search tool. J. Mol. Biol. 215, 403-410. doi: 10.1016/S00222836(05)80360-2
In general, a large number of human genes are regulated by bi-directional promoters (Trinklein et al., 2004) and highly active HERV promoters might serve as bi-directional promoters (Domansky et al., 2000). The chromosomal organization of the PRODH/ERVK-24 locus together with our expression data in GCT suggests that both genes are co-regulated. Interestingly, expression of $P R O D H$ in germ line cells is evolutionarily highly conserved since germ line stem cells from Drosophila express high amounts of the PRODH homolog sluggish $A$ (Kai et al., 2005). These data suggest that expression of $P R O D H$ is a feature of cells with an embryonic phenotype. The co-expression of ERVK-24 together with PRODH might be a consequence of the active chromatin state in GCT. Whether the expression of ERVK24 and PRODH has consequences for the tumor cell biology requires further investigation.

\section{CONCLUSION}

In addition to direct effects of HERV expression, co-regulation of neighboring genes should be considered as possible mechanism for HERV-associated diseases. This co-regulation can be associated with differentiation processes regulated by epigenetic mechanisms, as we have shown using GCT cell lines reflecting different stages of development.

\section{AUTHOR CONTRIBUTIONS}

TM and MS designed the study and wrote the paper. All authors performed experiments, analyzed the data, and approved the final version of the paper.

\section{FUNDING}

Our work was supported by the Wilhelm-Roux-Programm of the Medical Faculty of the Martin Luther University HalleWittenberg.

\section{ACKNOWLEDGMENTS}

We thank Franziska Reipsch for technical assistance.

\section{SUPPLEMENTARY MATERIAL}

The Supplementary Material for this article can be found online at: https://www.frontiersin.org/articles/10.3389/fmicb. 2018.01253/full\#supplementary-material

Andrews, P. W., Casper, J., Damjanov, I., Duggan-Keen, M., Giwercman, A., Hata, J., et al. (1996). Comparative analysis of cell surface antigens expressed by cell lines derived from human germ cell tumours. Int. J. Cancer 66, 806-816. doi: 10.1002/(SICI)1097-0215(19960611)66:6<806::AID-IJC17>3.0. $\mathrm{CO} ; 2-0$ 
Argaw-Denboba, A., Balestrieri, E., Serafino, A., Cipriani, C., Bucci, I., Sorrentino, R., et al. (2017). HERV-K activation is strictly required to sustain CD133+ melanoma cells with stemness features. J. Exp. Clin. Cancer Res. 36:20. doi: 10.1186/s13046-016-0485-x

Barbulescu, M., Turner, G., Seaman, M. I., Deinard, A. S., Kidd, K. K., and Lenz, J. (1999). Many human endogenous retrovirus K (HERV-K) proviruses are unique to humans. Curr. Biol. 9, 861-868. doi: 10.1016/S0960-9822(99)80390-X

Bender, H. U., Almashanu, S., Steel, G., Hu, C. A., Lin, W. W., Willis, A., et al. (2005). Functional consequences of PRODH missense mutations. Am. J. Hum. Genet. 76, 409-420. doi: 10.1086/428142

Boese, A., Sauter, M., Galli, U., Best, B., Herbst, H., Mayer, J., et al. (2000). Human endogenous retrovirus protein CORF supports cell transformation and associates with the promyelocytic leukemia zinc finger protein. Oncogene 19, 4328-4336. doi: 10.1038/sj.onc. 1203794

Boller, K., Schönfeld, K., Lischer, S., Fischer, N., Hoffmann, A., Kurth, R., et al. (2008). Human endogenous retrovirus HERVK113 is capable of producing intact viral particles. J. Gen. Virol. 89, 567-572. doi: 10.1099/vir.0.83534-0

Bronson, D. L., Andrews, P. W., Solter, D., Cervenka, J., Lange, P. H., and Fraley, E. E. (1980). Cell line derived from a metastasis of a human testicular germ cell tumor. Cancer Res. 40, 2500-2506.

Bronson, D. L., Andrews, P. W., Vessella, R. L., and Fraley, E. E. (1983). "In vitro differentiation of human embryonal carcinoma cells," in Teratocarcinoma Stem Cells, eds L. M. Silver, G. R. Martin, and S. Strickland (Cold Spring Harbor, NY: Cold Spring Harbor Laboratory Press), 597-605.

Casper, J., Schmoll, H. J., Schnaidt, U., and Fonatsch, C. (1987). Cell lines of human germinal cancer. Int. J. Androl. 10, 105-113. doi: 10.1111/j.1365-2605.1987. tb00171.x

Degenhardt, K., Mathew, R., Beaudoin, B., Bray, K., Anderson, D., Chen, G., et al. (2006). Autophagy promotes tumor cell survival and restricts necrosis, inflammation, and tumorigenesis. Cancer Cell 10, 51-64. doi: 10.1016/j.ccr. 2006.06.001

Diehl, V., Kirchner, H. H., Burrichter, H., Stein, H., Fonatsch, C., Gerdes, J., et al. (1982). Characteristics of Hodgkin's disease derived cell lines. Cancer Treat. Rep. $66,615-632$.

Domansky, A. N., Kopantzev, E. P., Snezhkov, E. V., Lebedev, Y. B., Leib-Mosch, C., and Sverdlov, E. D. (2000). Solitary HERV-K LTRs possess bi-directional promoter activity and contain a negative regulatory element in the $\mathrm{U} 5$ region. FEBS Lett. 472, 191-195. doi: 10.1016/S0014-5793(00)01460-5

Drexler, H. G., Gaedicke, G., Lok, M. S., Diehl, V., and Minowada, J. (1986). Hodgkin's disease derived cell lines HDLM-2 and L-428: comparison of morphology, immunological and isoenzyme profiles. Leuk. Res. 10, 487-500. doi: 10.1016/0145-2126(86)90084-6

Flockerzi, A., Ruggieri, A., Frank, O., Sauter, M., Maldener, E., Kopper, B., et al. (2008). Expression patterns of transcribed human endogenous retrovirus HERV-K(HML-2) loci in human tissues and the need for a HERV Transcriptome Project. BMC Genomics 9:354. doi: 10.1186/1471-2164-9-354

Galli, U. M., Sauter, M., Lecher, B., Maurer, S., Herbst, H., Roemer, K., et al. (2005). Human endogenous retrovirus rec interferes with germ cell development in mice and may cause carcinoma in situ, the predecessor lesion of germ cell tumors. Oncogene 24, 3223-3228. doi: 10.1038/sj.onc.1208543

Ge, X., Yamamoto, S., Tsutsumi, S., Midorikawa, Y., Ihara, S., Wang, S. M., et al. (2005). Interpreting expression profiles of cancers by genome-wide survey of breadth of expression in normal tissues. Genomics 86, 127-141. doi: 10.1016/j. ygeno.2005.04.008

Gogos, J. A., Santha, M., Takacs, Z., Beck, K. D., Luine, V., Lucas, L. R., et al. (1999). The gene encoding proline dehydrogenase modulates sensorimotor gating in mice. Nat. Genet. 21, 434-439. doi: 10.1038/7777

Hayward, D. C., Delaney, S. J., Campbell, H. D., Ghysen, A., Benzer, S., Kasprzak, A. B., et al. (1993). The sluggish-A gene of Drosophila melanogaster is expressed in the nervous system and encodes proline oxidase, a mitochondrial enzyme involved in glutamate biosynthesis. Proc. Natl. Acad. Sci. U.S.A. 90, 2979-2983. doi: 10.1073/pnas.90.7.2979

Kai, T., Williams, D., and Spradling, A. C. (2005). The expression profile of purified Drosophila germline stem cells. Dev. Biol. 283, 486-502. doi: 10.1016/j.ydbio. 2005.04.018

Kamesaki, H., Fukuhara, S., Tatsumi, E., Uchino, H., Yamabe, H., Miwa, H., et al. (1986). Cytochemical, immunologic, chromosomal, and molecular genetic analysis of a novel cell line derived from Hodgkin's disease. Blood 68, 285-292.
Lamprecht, B., Walter, K., Kreher, S., Kumar, R., Hummel, M., Lenze, D., et al. (2010). Derepression of an endogenous long terminal repeat activates the CSF1R proto-oncogene in human lymphoma. Nat. Med. 16, 571-579. doi: $10.1038 / \mathrm{nm} .2129$

Lemaître, C., Tsang, J., Bireau, C., Heidmann, T., and Dewannieux, M. (2017). A human endogenous retrovirus-derived gene that can contribute to oncogenesis by activating the ERK pathway and inducing migration and invasion. PLoS Pathog. 13:e1006451. doi: 10.1371/journal.ppat.1006451

Liu, W., Zabirnyk, O., Wang, H., Shiao, Y. H., Nickerson, M. L., Khalil, S., et al. (2010). miR-23b targets proline oxidase, a novel tumor suppressor protein in renal cancer. Oncogene 29, 4914-4924. doi: 10.1038/onc.2010.237

Liu, Y., Borchert, G. L., Surazynski, A., and Phang, J. M. (2008). Proline oxidase, a p53-induced gene, targets COX-2/PGE2 signaling to induce apoptosis and inhibit tumor growth in colorectal cancers. Oncogene 27, 6729-6737. doi: 10.1038 /onc.2008.322

Livak, K. J., and Schmittgen, T. D. (2001). Analysis of relative gene expression data using real-time quantitative PCR and the $2^{-\Delta \Delta C_{T}}$ Method. Methods 25, 402-408. doi: 10.1006/meth.2001.1262

Looijenga, L. H., Stoop, H., de Leeuw, H. P., de Gouveia Brazao, C. A., Gillis, A. J., van Roozendaal, K. E., et al. (2003). POU5F1 (OCT3/4) identifies cells with pluripotent potential in human germ cell tumors. Cancer Res. 63, 2244-2250.

Mayer, J., Blomberg, J., and Seal, R. L. (2011). A revised nomenclature for transcribed human endogenous retroviral loci. Mob. DNA 2:7. doi: 10.1186/ 1759-8753-2-7

Mueller, T., Mueller, L. P., Luetzkendorf, J., Voigt, W., Simon, H., and Schmoll, H.J. (2006). Loss of Oct-3/4 expression in embryonal carcinoma cells is associated with induction of cisplatin resistance. Tumour Biol. 27, 71-83. doi: 10.1159/ 000092324

Oosterhuis, J. W., and Looijenga, L. H. (2005). Testicular germ-cell tumours in a broader perspective. Nat. Rev. Cancer 5, 210-222. doi: 10.1038/nrc1568

Pera, M. F., Lafita, M. J. B., and Mills, J. (1987). Cultured stem-cells from human testicular teratomas: the nature of human embryonal carcinoma, and its comparison with two types of yolk-sac carcinoma. Int. J. Cancer 40, 334-343. doi: $10.1002 /$ ijc. 2910400309

Ruprecht, K., Ferreira, H., Flockerzi, A., Wahl, S., Sauter, M., Mayer, J., et al. (2008). Human endogenous retrovirus family HERV-K(HML-2) RNA transcripts are selectively packaged into retroviral particles produced by the human germ cell tumor line Tera-1 and originate mainly from a provirus on chromosome 22q11.21. J. Virol. 82, 10008-10016. doi: 10.1128/JVI.01016-08

Schaadt, M., Fonatsch, C., Kirchner, H., and Diehl, V. (1979). Establishment of a malignant, Epstein-Barr-virus (EBV)-negative cell-line from the pleura effusion of a patient with Hodgkin's disease. Blut 38, 185-190. doi: 10.1007/BF01007965

Sperger, J. M., Chen, X., Draper, J. S., Antosiewicz, J. E., Chon, C. H., Jones, S. B., et al. (2003). Gene expression patterns in human embryonic stem cells and human pluripotent germ cell tumors. Proc. Natl. Acad. Sci. U.S.A. 100, 13350-13355. doi: 10.1073/pnas.2235735100

Staege, M. S., Hansen, G., Baersch, G., and Burdach, S. (2004). Functional and molecular characterization of interleukin-2 transgenic Ewing tumor cells for in vivo immunotherapy. Pediatr. Blood Cancer 43, 23-34. doi: 10.1002/pbc. 20013

Sturn, A., Quackenbush, J., and Trajanoski, Z. (2002). Genesis: cluster analysis of microarray data. Bioinformatics 18, 207-208. doi: 10.1093/bioinformatics/18. 1.207

Sutherland, H. F., Wadey, R., McKie, J. M., Taylor, C., Atif, U., Johnstone, K. A., et al. (1996). Identification of a novel transcript disrupted by a balanced translocation associated with DiGeorge syndrome. Am. J. Hum. Genet. 59, 23-31.

Trinklein, N. D., Aldred, S. F., Hartman, S. J., Schroeder, D. I., Otillar, R. P., and Myers, R. M. (2004). An abundance of bidirectional promoters in the human genome. Genome Res. 14, 2-6.

Turner, G., Barbulescu, M., Su, M., Jensen-Seaman, M., Kidd, K. K., and Lenz, J. I. (2001). Insertional polymorphisms of full-length endogenous retroviruses in humans. Curr. Biol. 11, 1531-1535. doi: 10.1016/S0960-9822(01)00455-9

Vogelzang, N. J., Bronson, D., Savino, D., Vessella, R. L., and Fraley, E. F. (1985). A human embryonal-yolk sac carcinoma model system in athymic mice. Cancer 55, 2584-2593. doi: 10.1002/1097-0142(19850601)55:11<2584:: AID-CNCR2820551110>3.0.CO;2-B 
Winkler, C., Steingrube, D. S., Altermann, W., Schlaf, G., Max, D., Kewitz, S., et al. (2012). Hodgkin's lymphoma RNA-transfected dendritic cells induce cancer/testis antigen-specific immune responses. Cancer Immunol. Immunother. 61, 1769-1779. doi: 10.1007/s00262-012-1239-z

Wolf, J., Kapp, U., Bohlen, H., Kornacker, M., Schoch, C., Stahl, B., et al. (1996). Peripheral blood mononuclear cells of a patient with advanced Hodgkin's lymphoma give rise to permanently growing Hodgkin-Reed Sternberg cells. Blood 87, 3418-3428.

Zabirnyk, O., Liu, W., Khalil, S., Sharma, A., and Phang, J. M. (2010). Oxidized low-density lipoproteins upregulate proline oxidase to initiate ROS-dependent autophagy. Carcinogenesis 31, 446-454. doi: 10.1093/carcin/bgp299
Conflict of Interest Statement: The authors declare that the research was conducted in the absence of any commercial or financial relationships that could be construed as a potential conflict of interest.

Copyright (c) 2018 Mueller, Hantsch, Volkmer and Staege. This is an open-access article distributed under the terms of the Creative Commons Attribution License (CC BY). The use, distribution or reproduction in other forums is permitted, provided the original author(s) and the copyright owner are credited and that the original publication in this journal is cited, in accordance with accepted academic practice. No use, distribution or reproduction is permitted which does not comply with these terms. 